\title{
MECP2 et retard mental
}

Il aura fallu beaucoup de temps et d'efforts pour découvrir la cause moléculaire du syndrome de Rett, cette encéphalopathie extrêmement sévère qui n'atteint que les petites filles. Mais depuis la découverte de l'implication dans cette maladie du gène MECP2 (pour methyl-CpG-binding protein-2), les recherches entreprises, nombreuses désormais, commencent à porter leurs fruits.

Le gène $M$ ecp2 a été découvert chez le rat en 1993 [1]. II appartient à la famille des gènes codant pour des protéines possédant un domaine de fixation aux dinucléotides CpG méthylés (MBD). II fut retrouvé ensuite chez la souris, localisé sur le chromosome $X$ [2], puis chez I'homme où il est soumis à l'inactivation [3]. Par la suite, il fut démontré que s'il n'était pas indispensable à la multiplication des cellules souches embryonnaires (ES) mâles, il l'était en revanche pour le développement des embryons de souris mâles [4] .

MECP2 possède, en plus de son domaine MBD, un domaine de répression transcriptionnelle (TRD) pouvant fonctionner à distance in vitro et in vivo [5] (figure 1) et interagissant avec un complexe contenant un répresseur de transcription, $m S i n 3 A$, et une histone désacétylase (m/s 1997, n¹0, p. 1205). L'utilisation de trichostatine $A$, inhibitrice de la désacétylation, avait révélé que cette réaction est essentielle dans le mécanisme de répression. Avec ses domaines MBD et TRD, agissant sur la méthylation de l'ADN et la désacétylation des histones, la protéine MECP2, concentrée dans I'hétérochromatine péricentromèrique, agit sur le modelage de la chromatine et l'extinction à distance d'autres gènes qui restent encore à trouver. M ECP2 était le candidat idéal pour le syndrome de Rett [6] : létalité chez les garçons (comme chez les souriceaux

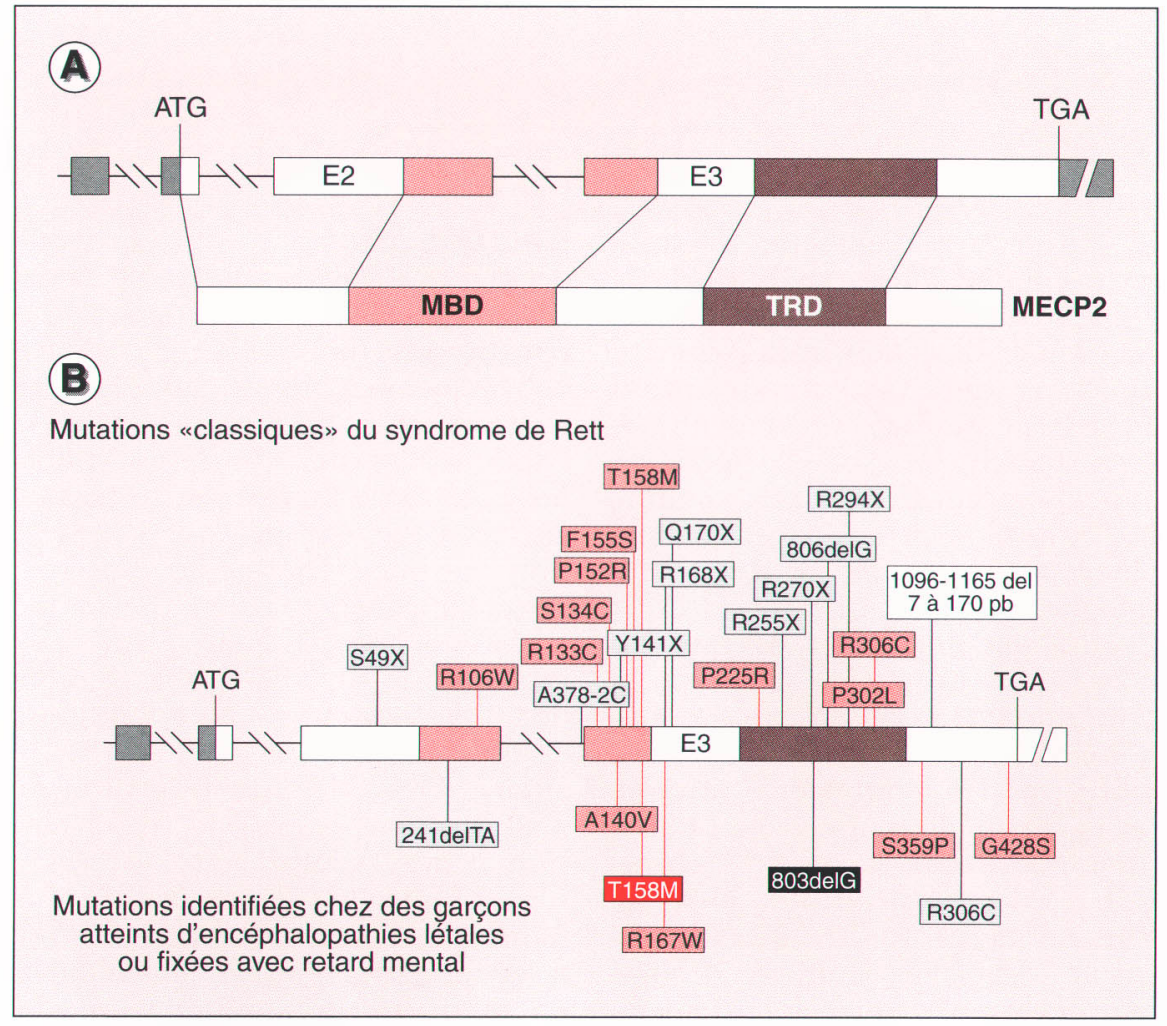

Figure 1. Gène MECP2 et retards mentaux. A. Représentation du gène MECP2 et de la protéine qui contient deux domaines fonctionnels MBD (methyl-CpG binding domain) et TRD (domaine de répression transcriptionnelle). B. Localisation des mutations de MECP2 dans le syndrome de Rett classique et dans les deux formes d'atteinte chez le garçon [8]. Les mutations faux sens sont en rose, et non sens en gris. Les mutations responsables d'encéphalopathies létales chez les garçons sont représentées en rouge (faux sens) et en noir.

mâles) et encéphalopathie sévère et progressive chez les filles, d'autant plus que le gène se situe en Xq28, dans la région où le locus du syndrome de Rett avait fini - à grand peine en raison de la raretés des cas familiaux - par être localisé. Encore fallait-il le démontrer, ce qui fut fait en 1999 par une équipe américaine ([7] et m/s 1999, n¹1, p. 1334). Depuis, l'étude de grandes cohortes de filles atteintes de syndrome de
Rett a confirmé le rôle de M ECP2 et a permis de répertorier une dizaine de mutations [8]. Le pourcentage de détection des mutations se situe aux alentours de $80 \%$. Celles-ci surviennent de novo dans la plupart des cas et I'X muté est le plus souvent d'origine paternelle. Chez les filles atteintes, l'inactivation de I'X est aléatoire ( $90 \%$ des cas environ). Toutefois, dans certains cas familiaux, une origine maternelle est 
retrouvée, et s'explique par une inactivation préférentielle de I'X porteur d'une mutation de MECP2 chez des femmes qui ne présentent alors peu ou pas de symptômes. Mais lorsqu'il est transmis à leurs filles - qui ont une inactivation au hasard de leur $X$ - le chromosome X porteur de la mutation entraînera les manifestations caractéristiques du syndrome de Rett.

Tout récemment, deux modèles murins ont été étudiés. Ils donnent un aperçu du retentissement des mutations de MECP2 sur le fonctionnement cérébral.

Une équipe américaine a obtenu deux modèles murins: des souris avec absence de $M$ ecp2 ( $M$ exp2 ${ }^{-1-}$ ) et des souris avec une mutation conditionnelle dans le cerveau ( $p$ ar le système Cre-loxp) [9]. Les deux modèles murins ont le même phénotype, avec la même évolution : les souris mâles sont normales jusqu'à la cinquième semaine, puis présentent des tremblements, des troubles respiratoires et comportementaux. Après une période d'obésité et d'hypoactivité, leur état général se dégrade et elles meurent environ vers la dixième semaine. Les femelles hétérozygotes sont normales durant les quatre premiers mois de vie, puis elles prennent du poids et présentent des troubles de la coordination motrice. $L$ 'autopsie révèle une réduction sensible du poids du cerveau et de la taille des neurones, mais pas de signes de neurodégénérescence. Si la délétion de M ecp2 est reportée à la période post-natale, on observe le même phénotype, mais d'apparition un peu plus tardive.

Une équipe anglaise, par des techniques similaires, a obtenu des souris homo- $M$ ecp2 $2^{-1-}$ ou hémizygotes $M$ ecp $2^{-1 y}$ [10]. Celles-ci sont viables et fertiles. Si leur développement, en particulier le poids corporel, varie selon les lignées de souris utilisées, il n'existe en revanche pas de différence sur le plan neurologique. Les souris M ecp2 nul apparaissent normales à la naissance et dans les semaines qui suivent puis, entre 3 et 8 semaines, on observe une réduction des mouvements, avec incoordination motrice, contracture des pattes de derrière et logique des différents tissus, le cerveau en particulier, n'a montré aucune anomalie. La mort survient en général vers le $54^{\mathrm{e}}$ jour. Les femelles hétérozygotes, qui semblent normales pendant plusieurs mois, finissent elles aussi par présenter des troubles du comportement.

Bien que les résultats ne soient pas absolument superposables, ces deux études démontrent que, contrairement à ce qui avait été publié précédemment [4], le gène M ecp2 n'est pas indispensable au développement embryonnaire et que I'absence d'expression de M ecp2 ne retentit pas sur le développement cérébral. Le phénotype qui apparaît chez les souris est moins sévère que chez I'homme, puisque les mutants mâles survivent jusqu'à l'âge adulte. II ressemble cependant beaucoup aux manifestations cliniques du syndrome de Rett qui, on le sait, n'apparaissent que vers l'âge de six mois. II semble donc que la perte d'expression de MECP2 retentit essentiellement sur la physiologie des neurones post-mitotiques et que le trouble est cellule-autonome, ce qui expliquerait le ralentissement de la croissance céphalique chez les filles par atteinte sélective des neurones dont le $X$ muté est actif.

Le gène MECP2 étant ubiquitaire et devant agir comme répresseur transcriptionnel dans toutes les cellules, il est surprenant de constater que son inactivation a pour seul retentissement une atteinte cérébrale spécifique.

Mais il y a plus surprenant encore. Contrairement à ce que l'on avait cru jusqu'à présent, on découvre des mutations du gène MECP2 chez des garçons. Elles se manifestent par deux formes syndromiques différentes: 1) l'une correspond à une encéphalopathie convulsivante avec décès précoce, pressentie dès 1998, avant même la découverte du gène grâce à quelques cas familiaux [11] ; 2) la seconde est beaucoup plus inattendue. II s'agit d'un retard mental compatible avec une survie à l'âge adulte. Dans les cas familiaux, les femmes qui transmettent la maladie ont une activation aléatoire de I'X, mais ne présentent que peu ou pas de symptômes. On retrouve aussi des formes sporadiques qui sont jusqu'à présent sousévaluées en raison de l'absence de critères spécifiques $[12,13]$.

II convient donc de vérifier l'intégrité de M ECP2 chez les garçons porteurs de retards mentaux pour lesquels les recherches d'autres étiologie ( $X$ fragile, syndrome d'Angelman, entre autres) se sont avérées négatives, afin de pouvoir établir éventuellement une corrélation génotype-phénotype. Dès à présent, il semble que les mutations dans cette forme non spécifique chez le garçon soient différentes des mutations des formes de syndrome de Rett classique. Elles n'altéreraient que partiellement la fonction de MECP2 (en dehors des domaines MBD et TRD) (figure 1). Mais ceci mérite encore d'autres études.

Le gène MECP2, qui, comme RSK2 impliqué dans une autre encéphalopathie progressive, le syndrome de Coffin Lowry (m/s 1997, $\left.n^{\circ} 1, p .107\right)$, intervient dans le remodelage chromatinien, a donc encore beaucoup à nous apprendre sur les mécanismes d'activation ou d'extinction des gènes.

1. Lewis JD, Meehan RR, Henzel WJ, et al. Purification, sequence, and cellular localisation of a novel chromosomal protein that binds to methylated DNA. Cell 1992; 69: 905-14.

2. Quaderi NA, Meehan RR, Tate PH, et al. Genetic and physical mapping of a gene encoding a methyl CPG binding protein, Mecp2, to the mouse X chromosome. Genomics 1994; 22: 64851.

3. D'Esposito M, Quaderi NA, Ciccodicola A, et al. Isolation, physical mapping, and Northern analysis of the $X$-linked human gene encoding methylCpG-binding protein, MECP2. Mammalian Genome 1996; 7: 533-5.

4. Tate P, Skarnes W, Bird A. The methyl-CpG binding protein $\mathrm{MeCP} 2$ is essential for embryonic development in the mouse. $N$ at Genet 1996; 12 : 205.

5. Nan X, Campoy FJ, Bird A. MeCP2 is a transcriptional repressor with abundant binding sites in genomics chromatin. Cell 1997; 88: 471-81. 6 . Willard HF, Hendrich BD . Breaking the silence in Rett syndrome. Nat Genet $1999 ; 23: 127-8$.

7. Amir RE, Van den Veyver IB, Wan M, Tran CQ, Francke U, Zoghbi HY. Rett syndrome is caused by mutations in $X$-linked MECP2, encoding methyl-CpG-binding protein 2. Nat Genet 1999; 23: 185-8.

8. Bourdon V, Philippe C, Labrune O, Amsallem $D$, Arnould $C$, Jonveaux P. A detailed analysis of the MECP2 gene: prevalence of recurrent mutations and gross DNA rearrangements in Rett syndrome patients. H um G enet 2001; 108: 43-50.

9. Chen RZ, Akbarian S, Tudor M, Jaenisch R. Deficiency of methyl-CpG binding protein-2 in 
CNS neurons results in a Rett-like phenotype in mice. Nat Genet 2001; 27: 327-31.

10. Guy J, Hendrich B, Holmes M, Martin JE, Bird A. A mouse Mecp2-null mutation causes neurological symptoms that mimic Rett syndrome. Nat Genet $2001 ; 27: 322-6$

11. Schanen NC, Kurczynski TW, Brunelle D, Woodcock MM, Dure LS, Percy ALK. Neonatal encephalopathy in two boys in families with recurrent Rett syndrome. J Child Neurol 1998; 13: 229 31.

12. Meloni I, Bruttini M, Longo I, et al. MECP2 mutations in Rett syndrome gene, MECP2, causes $X$-linked mental retardation and progressive spasticity in males. Am J H um Gen et 2000; 67: 982-5. 13. Orrico A, Lam C-W, Galli L, et al. MECP2 mutation in male patients with non-specific $X$-linked mental retardation FEBS 2000 ; 24106 : 1-4.

\section{Simone Gilgenkrantz}

9, rue Basse, 54330 Clérey-sur-Brenon, France.

\section{Violaine Bourdon}

Laboratoire de génétique médicale, UPRES-INRA 952, CHRU, rue du M orvan, 54511 Vandœu vre-les-Nancy, France.

\section{BRÈVES}

Rétrovirus et schizophrénie, quand pol s'exprime. C'est à l'aboutissement d'une longue quête, puisqu'elle a commence il y a plus de 15 ans [1], qu'une équipe américano-allemande menée par Hakan Karlsson (Johns Hopkins, Baltimore USA) nous invite en démontrant la présence de séquences proches du gène rétroviral $\mathrm{pol}$ dans le liquide céphalo-rachidien de patients atteints de schizophrénie. Près de $30 \%$ des 35 patients testés, atteints d'une schizophrénie d'apparition récente, présentaient en effet ces séquences, alors que ce n'était le cas d'aucun des 52 témoins (et d'un seul patient schizophrène chronique sur 20). Ces séquences correspondaient, plus particulièrement, à celles rencontrées dans la famille des rétrovirus humains endogènes HERV-W. Une étude post-mortem parallèle a permis aux auteurs de confirmer une augmentation de la transcription de rétrovirus de la même famille dans la région frontale du cerveau d'autres patients schizophrènes. D'une façon intéressante, les auteurs ont observé dans les séquences pol retrouvées chez les patients des codons stop qui semblaient devoir interdire la réplication des virus, ce qui suggère une synthèse endogène plutôt qu'une source exogène. II est clair - et les auteurs ne le prétendent sûrement pas - que la schizophrénie n'est pas une maladie mono-factorielle. Les études de familles ont depuis longtemps démontré, notamment, le rôle de facteurs génétiques (toutefois encore mal determinés). L'existence d'un élément rétroviral, qui agirait essentiellement lors du déclenchement de la maladie, semble donc mis à l'ordre du jour. En dehors de l'intérêt général que présentent ces résultats - dont on attend néanmoins la réplication, I'histoire des recherches étiopathogéniques des maladies psychiatriques étant riche en fausses bonnes nouvelles - ils pourraient déboucher sur des applications thérapeutiques, le traitement de la schizophrénie passant alors, dans un curieux détour, par la pharmacopée antivirale.
[1. Crow T]. Br J Psychiatry 1984; $145: 243-53$.

[2. Karlsson $\mathrm{H}$, et al. Proc Natl Acad Sci USA 2001; 98 : 4634-9.]

Des cellules progénitrices amplifiées à partir du cerveau adulte... chez l'homme aussi. Dans une courte lettre adressée à Nature, Fred Gage et son équipe (Salk Institute, CA, USA) concluent une quête de près de 10 ans en démontrant que le cerveau adulte de I'homme contient des cellules capables de proliférer in vitro et de donner naissance à des neurones [1]. De telles cellules avaient été observées chez la souris par l'équipe de Samuel Weiss (Université de Calgary, Canada) en 1992 [2], et l'équipe de Fred Gage avait, il y a trois ans, rapporté l'existence d'une neurogenèse dansl'hippocampe adulte chez I'homme [3]. La boucle est bouclée, ce qui vaut pour la souris vaut pour l'homme: des cellules souches adultes peuvent être amplifiées in vitro, dans des conditions de culture particulières nécessitant de fortes concentrations de FGF-2 et un cofacteur, la forme glycosylée de la cystatine $C$. Incidemment, ces travaux montrent que les limites apparentes des capacités de ces cellules souches identifiées chez la souris adulte valent aussi pour l'homme: seulement $1 \%$ à $1 \%$ des cellules différenciées sont des neurones, et plus l'âge du cerveau dans lequel les cellules souches sont prélevées est avancé, plus petit est le nombre de passages possible avant l'entrée en sénescence. On attend à présent les mises au point qui permettront de contourner ces problèmes et de créer, à partir de ce matériel, les banques de cellules implantables dont tout le monde rêve.

[1. Palmer TD, et al. Nature 2001; 411: 42-3.]

[2. Reynolds BA, Weiss S. Science 1992; 255: 1707-10.]

[3. Eriksson PS, et al. Nat M ed 1998; 4: 1313-7.] 